\title{
Intermittency, local isotropy, and non-Gaussian statistics in atmospheric surface layer turbulence
}

\author{
Gabriel G. Katul \\ School of the Environment, Box 90328, Duke University, Durham, North Carolina 27708-0328 \\ Marc B. Parlange \\ Hydrologic Science, University of California, Davis, Davis, California 95616 and Department of \\ Agricultural and Biological Engineering, University of California, Davis, Davis, California 95616 \\ Chia R. Chu \\ Department of Civil Engineering, National Central University, Chung-Li, Taiwan
}

(Received 26 May 1993; accepted 8 March 1994)

\begin{abstract}
Orthonormal wavelet expansions are applied to atmospheric surface layer velocity and temperature measurements above a uniform bare soil surface that exhibit a long inertial subrange energy spectrum. In order to investigate intermittency effects on Kolmogorov's theory, a direct relation between the $n$ th-order structure function and the wavelet coefficients is derived. This relation is used to examine deviations from the classical Kolmogorov theory for velocity and temperature in the inertial subrange. The local nature of the orthonormal wavelet transform in physical space aided the identification of events directly contributing to intermittency buildup at inertial subrange scales. These events occur at edges of large eddies and contaminate the Kolmogorov inertial subrange scaling. By suppressing these events, the statistical structure of the inertial subrange for the velocity and temperature, as described by Kolmogorov's theory, is recovered. The suppression of intermittency on the $n$ th-order structure function is carried out via a conditional wavelet sampling scheme. The conditioned wavelet statistics reproduced the Kolmogorov scaling (up to $n=6$ ) in the inertial subrange and result in a zero intermittency factor. The conditional wavelet statistics for the mixed velocity temperature structure functions are also presented. It was found that the conditional wavelet statistics for these mixed moments result in a thermal intermittency parameter consistent with other laboratory and field measurements. The relationship between Kolmogorov's theory and near-Gaussian statistics for velocity and temperature gradients is also considered.
\end{abstract}

\section{INTRODUCTION}

From Kolmogorov ${ }^{1}$ theory (K41), the ensemble average of the $n$th order velocity difference $\left(\Delta u_{i}\right)$ between two points separated by spatial distance $(r)$, in the inertial subrange, can be computed using

$$
\left\langle\left|\Delta u_{i}\right|^{n}\right\rangle=K_{n}(\langle\epsilon\rangle)^{n / 3} r^{n / 3},
$$

where $\epsilon$ is the turbulent energy dissipation rate

$$
\epsilon=\frac{\nu}{2}\left(\frac{\partial u_{i}}{\partial x_{j}}+\frac{\partial u_{j}}{\partial x_{i}}\right)^{2}
$$

and $u_{i}$ are the velocity components $(i=1,2,3), \nu$ is the kinematic viscosity, $K_{n}$ is a universal constant independent of the flow but depends on $n, n$ is the order-of-the-structure function, $r$ is the separation distance that is much smaller than the integral length scale $(L)$ but much larger than the Kolmogorov microscale $\eta\left(=\left[\nu^{3} /\langle\epsilon\rangle\right]^{1 / 4}\right)$, and $\langle\cdot\rangle$ is the ensemble averaging operator.

The scaling laws in (1) have been applied to scalars such as temperature, humidity, carbon dioxide concentrations, as well as many other flow variables, and (1) was found to hold by many experiments for $n=2$ (see Ref. 2, pp. 453-527). However, (1) appears to be less accurate for $n>2$ as evidenced by many other laboratory experiments. ${ }^{3}$ Deviations from (1) have been attributed to intermittency buildup within the inertial subrange, as originally noted by Landau and Lifshitz (see Refs. 4 and 5 for a different interpretation). As a result, many phenomenological models and intermittency corrections to K41 have been proposed. These corrections include intermittency effects intrinsic to the dissipation rate or fractal-like buildup of intermittency during the energy cascade process. ${ }^{6}$ Examples of these phenomenological models include the $\beta$ model, ${ }^{7}$ the lognormal model, ${ }^{8}$ and other multifractal models., 90,11 Kuznetsov $^{12}$ classified the intermittency from these types of models as "internal," since the variability in the instantaneous dissipation rate is only considered. Using high Reynolds number turbulent velocity measured in a wind tunnel, they showed that "external" intermittency plays a key role in the energy cascade. External intermittency arises due to the direct correlation between the large-scale motion and the inertial subrange scales. It is not yet established whether external intermittency is a property of the Navier-Stokes equations or the result of boundary conditions. However, we should note that the large-scale eddy motion cannot be independent of the boundary conditions.

Many atmospheric surface layer (ASL) flow spectra exhibit an inertial subrange that extends over many decades so that intermittency effects on K41 and the energy cascade become important. ${ }^{13.2}$ The turbulence in the ASL is ideal for investigating K41 and intermittency effects on K41, since the Reynolds number is very high, and the scale separation between $L$ and $\eta$ is large $\left(L / \eta \approx 10^{6}\right.$ for ASL turbulent flows). Intermittency studies in the natural environment can encounter difficulties due to (i) the limited sampling period over 
which steady state mean meteorological conditions exist, (ii) the need for instrumentation that is free of atmospheric contamination and possible temperature and humidity calibration drifts, and (iii) the requirement for instrumentation that is field robust and capable of providing all three velocity components since changes in wind direction are inevitable.

As discussed in Katul, ${ }^{14}$ the first difficulty limits the number of data points that can be used to evaluate the ensemble average in (1). Typically, the ergodic hypothesis is used to evaluate the ensemble average in (1) from measured time averages. ${ }^{15}$ The convergence of time averages to ensemble averages requircs a very large number of measurements, especially as the power $n$ increases. The large number of measurements may not always be attainable in many field studies primarily due to unsteadiness in the mean meteorological conditions. The second and third difficulties limit the use of many fast response sensors that can resolve scales as small as $\eta$, such as hot wire probes.

The development of analyzing tools that allow the study of intermittency effects in the ASL from limited number of field measurements is therefore necessary. The purpose of this paper is to investigate the usefulness of orthonormal wavelet transforms in quantifying intermittency effects on K41 using ASL velocity and temperature measurements. The wavelet transform is applied to $21 \mathrm{~Hz}$ triaxial ultrasonic anemometer velocity and temperature measurements above a large and uniform bare soil surface. Since intermittency investigations typically utilize Fourier power spectra and structure functions, we establish a relation between the wavelet coefficients and these statistical measures. We also use conditional wavelet statistics that are developed to isolate events directly causing intermittency buildup in the inertial subrange. A brief review of wavelet transforms with emphasis on applications to turbulence measurements is presented.

\section{ANALYSIS OF TURBULENCE MEASUREMENTS USING WAVELET TRANSFORMS}

In this section, a brief review of wavelet transform theory that is relevent to this study is presented. Wavelet transforms are recent mathematical tools based on group theory and square integrable functions that unfold turbulence signals into space and scale. Continuous wavelet transforms have been applied to many turbulence measurements and proved to be successful in identifying local scaling exponents, ${ }^{16-20}$ intermittency visualization, ${ }^{21}$ identification of ensemble coherent events for proper orthogonal decomposition investigations, ${ }^{22}$ and identification of coherent structures above and within canopies. ${ }^{23}$ Orthonormal wavelets are the discrete counterpart of continuous wavelets; however, they have the added feature of forming a complete basis with analyzing wavelet functions orthogonal to their translates. ${ }^{24,25,26}$ The application of orthonormal wavelets has added an important new techniques in the study and analysis of turbulence measurements. ${ }^{27-32,14}$ For completeness, a brief review of continuous and orthonormal wavelet transforms is given.

Analogous to Fourier transforms, wavelet transforms can be classified as either continuous or discrete. The continuous wavelet transform is first considered followed by a motivation for using discrete wavelet transform.

\section{A. Continuous wavelet transforms}

As shown by Grossmann, ${ }^{33}$ the continuous wavelet transform $W(b, a)$ of a real-square integrable signal $f(x)$ [i.e., $\int_{-\infty}^{+\infty} f(x)^{2} d x<\infty$ ] with respect to a real integrable analyzing wavelet $\psi(x)$ can be defined as

$$
W(b, a)=C_{g}^{1 / 2} \frac{1}{\sqrt{a}} \int_{-\infty}^{+\infty} \psi\left(\frac{t-b}{a}\right) f(t) d t,
$$

where $a$ is a scale dilation, $b$ is a position translation, and $C_{g}$ is defined by

$$
C_{g}=\int_{-\infty}^{+\infty}|K|^{-1}\left|\psi^{*}(K)\right|^{2} d K<\infty
$$

where $K$ is the wave number and $\psi^{*}$ is the Fourier transform of $\psi(x)$ given by

$$
\psi^{*}(K)=\int_{-\infty}^{+\infty} \psi(t) e^{-i K t} d t
$$

The continuous wavelet transform is commonly viewed as a numerical microscope whose optics, magnification, and position are given by $\psi(x), a$, and $b$, respectively. ${ }^{21}$ In order to classify as a wavelet, the function $\psi(x)$ has to satisfy the following conditions: (1) The admissibility condition, which requires that

$$
\int_{-\infty}^{+\infty} \psi(y) d y=0 .
$$

Simply stated, (6) requires that the average of $\psi(x)$ be zero. (2) The similarity condition, which requires the scale decomposition to be obtained by translation and dilation of onc analyzing function. (3) The invertibility condition, which requires at least one reconstruction formula for recovering $f(x)$ from its wavelet coefficients. The function $f(x)$ may be retrieved from the wavelet coefficients by

$$
f(x)=C_{g}^{-1 / 2} \int_{0}^{+\infty} \int_{-\infty}^{+\infty} a^{-1 / 2} \psi\left(\frac{x-b}{a}\right) W(a, b) \frac{d b d a}{a^{2}} .
$$

Further details regarding the wavelet transform theory can be found in many references (see, e.g., Refs. 25, 26, and 3436).

\section{B. Orthonormal wavelet expansions}

For the analysis of turbulence measurements, discrete wavelet transforms are preferred, since $f(x)$ is typically known at only discrete points $x_{j}$ (whose spacing depends on the resolution of the sensor and the sampling frequency). Therefore, it becomes necessary to discretize the scale (a) and the space (b) domain of (3). If $f\left(x_{j}\right)$ is defined by $N$ discrete points, one may consider simply discretizing the space domain of (3) by $N$ nodes and the scale domain of (3) by $N$ nodes (i.e., discretized by a series of Dirac-delta functions). In this manner, the wavelet transform of $f\left(x_{j}\right)$ re- 
quires $N^{2}$ wavelet coefficients. This discretization forms an over-complete description of $f\left(x_{j}\right)$ in the wavelet domain. Therefore, redundant information is injected by the wavelet transform of $f\left(x_{j}\right)$ that may or may not be advantageous depending on the specific application or purpose. For example, if some statistical analysis is to be performed on the wavelet coefficients, then some correlation may exist between the wavelet coefficients because of the transformation rather than the turbulence mechanism under consideration. To eliminate this redundancy, it is necessary to construct a complete and orthogonal wavelet basis. In this case, $N$ wavelet coefficients are necessary to describe $f\left(x_{j}\right)$ in the wavelet domain rather than $N^{2}$. Therefore, orthonormal wavelet transforms are suited for this purpose, since the basis functions are orthogonal and the mutual independence of the wavelet coefficients is guaranteed. As shown by Daubechies, ${ }^{35}$ Mallat, ${ }^{37,38}$ and Meyer ${ }^{24}$ using a logarithmic uniform spacing for the scale discretization with increasingly coarser spatial resolution at larger scales, a complete orthogonal wavelet basis can be constructed that allows the decomposition of $f\left(x_{j}\right)$ from $N$ wavelet coefficients. Note, unlike Fourier transforms, many wavelet basis functions are available for this decomposition. We choose the Haar wavelet basis for its differencing characteristics, since we are interested in developing explicit relations between the $n$ thorder structure function in (1) and the wavelet coefficients. The Haar basis $\psi(x)=\left(a^{-1 / 2}\right) \psi[(x-b) / a]$, where $a=2^{m}$ and $b=2^{m} i$ for $i, m \in \mathbf{Z}$, is given by

$$
\psi(x)=\left[\begin{array}{cc}
1 & \text { for } 0<x<1 / 2 \\
-1 & \text { for } 1 / 2 \leqslant x<1 \\
0 & \text { elsewhere }
\end{array}\right]
$$

where $i$ and $m$ are position and scale indices, respectively. As shown by Beylkin, ${ }^{39,40}$ for the Haar basis function, the wavelet coefficients $W T^{(m+1)}(k)$ and the coarse grained signal $S^{(m+1)}(k)$ (i.e., a low pass filtered signal) at scale $m+1$ can be determined from the signal $S^{(m)}$ at scale $m$ using

$$
\begin{aligned}
& W T^{(m+1)}(i)=\frac{1}{\sqrt{2}}\left[S^{(m)}(2 i-1)-S^{(m)}(2 i)\right], \\
& S^{(m+1)}(i)=\frac{1}{\sqrt{2}}\left[S^{(m)}(2 i-1)+S^{(m)}(2 i)\right]
\end{aligned}
$$

for $m=0$ to $M-1, i=0$ to $2^{M-m-1}-1$, and $M=\log _{2}(N), N$ is the number of samples (integer power of 2). For the Haar wavelet, the coarse grained signal defined by $(10)$ is a lowpass filtered function obtained by a simplc block average (see Daubechies ${ }^{35}$ for other types of filters). Hence, from (9) and (10), the wavelet coefficients and coarse grained signal may be calculated using the following pyramidal algorithm for a signal stored in vector $S^{(0)}$.

(1) Beginning with $m=0$, use (9) and (10) to calculate $S^{(1)}$ and the wavelet coefficients $W T^{(1)}$ at the first scale by looping over $i$ from 0 to $2^{M-1}-1$. This results in $S$ and $W T$ vectors each of length $N / 2$.

(2) Repeat step 1 with $m=1$ to calculate the next coarser scale's pair of vectors $S^{(2)}$ and $W T^{(2)}$ (each of length $N / 4$ ) from $S^{(1)}$.
(3) Repeat for larger scalc $m$ up to $M-1$ to produce a series of $S$ and $W T$ vectors of progressively decreasing length. Note that at $m=M-1$ the coarse grained signal converges to a point. This algorithm will yield $N-1$ wavelet coefficients defining the orthonormal Haar wavelet transform of the measured turbulence signal. The above pyramidal procedure, which is known as fast wavelet transforms (FWT), requires about $N$ computations vis-à-vis the $N \log _{2} N$ computational steps for fast Fourier transforms (FFT). The $N-1$ discrete Haar wavelet coefficients also satisfy the conservation of energy condition

$$
\sum_{j=0}^{j=N-1} f(j)^{2}=\sum_{m=1}^{m=M} \sum_{i=0}^{i=\left(2^{M-m}-1\right)}\left[W T^{(m)}(i)\right]^{2} .
$$

Equation (11) states that the sum of the square of the wavelet coefficients for all scales and positions conserves the norm of the signal. ${ }^{14,32}$ This is similar to Parseval's identity in Fourier expansions. ${ }^{26}$

\section{EXPERIMENT}

The data presented here were collected during an experiment in 22 August 1993 at 1:55 pm over a uniform bare soil surface at the University of California, Davis Campbell Tract facility. The field site is a Yolo clay loam soil contained within a larger site that extends uniformly some $250 \mathrm{~m}$ in all directions. The longitudinal $(U)$, lateral $(V)$, and vertical $(W)$ velocity components were measured at $z=2.0 \mathrm{~m}$ using a triaxial ultrasonic anemometer (Gill Instruments/1012R2) to an accuracy of $\pm 1 \%$. Sonic anemometers achieve their frequency response by sensing the effect of wind on transit times of sound pulses traveling in opposite directions across a known path length $d_{\mathrm{s} 1}(=0.149 \mathrm{~m}$ for the Gill sonic anemometer). The sonic anemometer is suited for these experiments since it is free of calibration nonlinearities and atmospheric contamination drifts. The main disadvantage of sonic anemometers is typically attributed to the wave-number distortion due to averaging over $d_{\mathrm{sl}}$. This distortion is generally restricted to wave numbers larger than $2 \pi / d_{\mathrm{sl}}\left(=42.2 \mathrm{~m}^{-1}\right)$ as discussed in Wyngaard ${ }^{41}$ and Friehe. ${ }^{42}$

The absolute air temperature $(T)$ was determined from the measured speed of sound $(c)$ fluctuations using $T=\alpha c^{2} / R_{d}$, where $\alpha=C_{p} / C_{v}(\approx 1.4), C_{p}$ and $C_{v}$ are the specific heat capacities of dry air under constant pressure and volume, respectively, and $R_{d}$ is the gas constant for dry air. $^{41,43}$ The influence of humidity variation on temperature was neglected. A comparison between the temperature determined from the triaxial sonic anemometer and temperature fluctuation measured from a fine wire chromel constantan thermocouple $(0.0127 \mathrm{~mm})$ is shown in Fig. 1(a). The thermocouple (TC) of Fig. 1(a) was placed at the same height as the sonic anemometer $(z=2.4 \mathrm{~m})$ but $60 \mathrm{~cm}$ away. The sampling frequency used in this comparison was $10 \mathrm{~Hz}$ and the sampling period was $13.65 \mathrm{~min}$. The main temperature structures are well captured by both instruments. The observed standard deviations of the thermocouple and sonic anemometer temperature measurements were nearly identical $(0.84$ and $0.82^{\circ} \mathrm{C}$, respectively). In Fig. 1(b), a comparison be- 

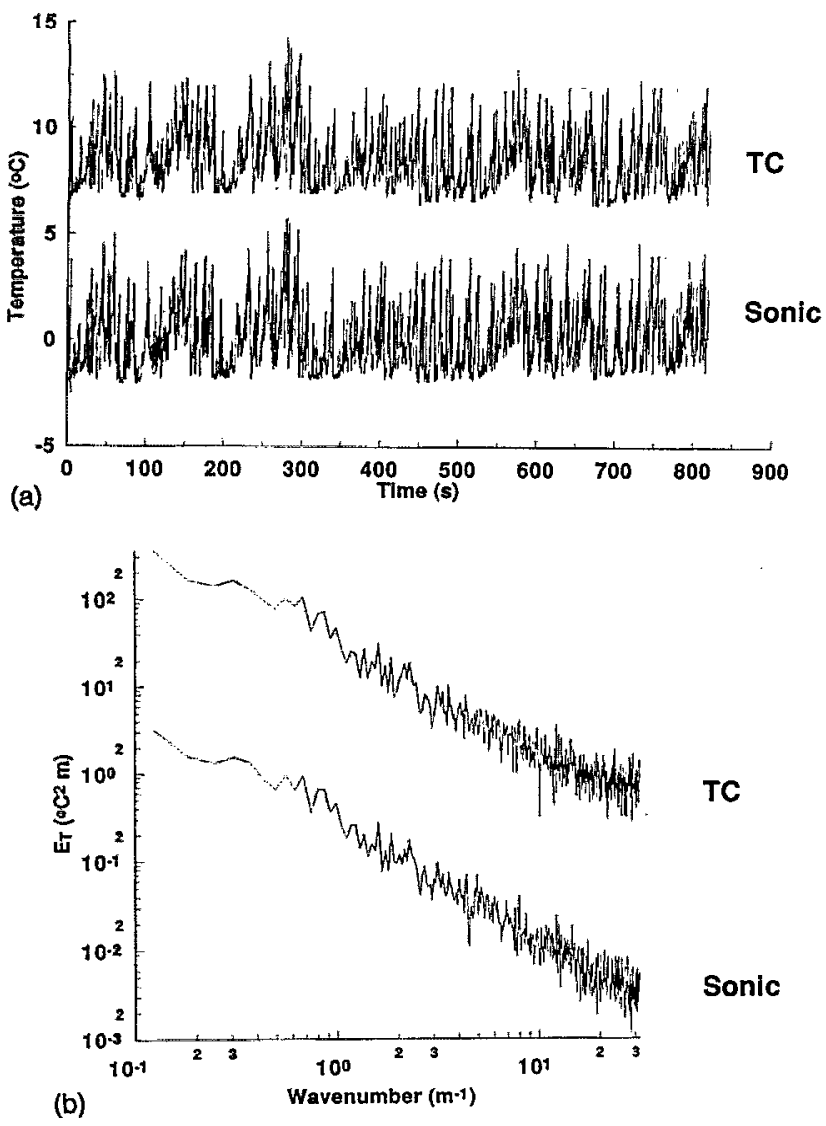

FIG. 1. (a) Comparison between thermocouple (TC) and triaxial sonic anemometer temperature measurements. The TC time series is shifted by $7.5^{\circ} \mathrm{C}$ to permit comparison. (b) Comparison between the thermocouple (TC) and triaxial sonic anemometer temperature spectra. The TC power spectrum is shifted by two decades to permit comparison at the high wavenumber end.

tween the power spectra of the two temperature time series is shown. The TC spectrum is shifted by two decades along the ordinate axis to permit comparison at the high wave-number end. At the low wave-number end of the spectra, the two sensors are in excellent agreement. The temperature spectrum from the thermocouple appears to "level off" at high wave numbers due to the limited resolution of the thermocouple. ${ }^{44}$ This suggests that the sonic anemometer has better fine-scale resolution than the TC for temperature measurements.

The sampling frequency $\left(f_{s}\right)$ of the sonic anemometer was $21 \mathrm{~Hz}$ and the sampling period $\left(T_{p}\right)$ was $26 \mathrm{~min}$. The short sampling period was necessary for steady state in the mean meteorological conditions. For $f_{s}=21 \mathrm{~Hz}$ and $T_{p}=26$ min, 32768 points, for each velocity component and speed of sound, were obtained (i.e., $N=32768$ ). A summary of the mean metcorological and turbulence conditions is presented in Table I. From Table I, the stability parameter $z / L_{\mathrm{MO}}$ is -0.44 indicating that buoyant and mechanical turbulent production are equally important for this study. We assume in this study that the velocity and temperature measurements can be decomposed, without ambiguity, into a mean and a fluctuating part.

The ratio of the root-mean-square (RMS) velocity

TABLE I. Summary of meteorological, turbulence, and surface roughness conditions during the experiment. The friction velocity was measured by the triaxial sonic anemometer. The momentum roughness length was determined in Ref. 32.

\begin{tabular}{ll}
\hline \multicolumn{2}{c}{ Meteorological conditions } \\
\hline Mean horizontal wind speed $(\langle U\rangle)$ & $2.44 \mathrm{~m} \mathrm{~s}^{-1}$ \\
Mean air temperature $\left(T_{a}\right)$ & $36.68^{\circ} \mathrm{C}$
\end{tabular}

Turbulence conditions

\author{
Friction Velocity $\left(u_{*}\right)$ \\ Sensible heat flux \\ RMS temperature $\left(\sigma_{T}\right)$ \\ RMS velocity $\left(\sigma_{w}\right)$ \\ RMS velocity $\left(\sigma_{u}\right)$
}

$$
\begin{aligned}
& 0.16 \mathrm{~m} \mathrm{~s}^{-1} \\
& 97 \mathrm{~W} \mathrm{~m}^{-2} \\
& 0.67^{\circ} \mathrm{C} \\
& 0.29 \\
& 1.20 \mathrm{~m} \mathrm{~s}^{-1}
\end{aligned}
$$

Atmospheric stability conditions

Height above ground surface $(z)$ Obukhov length $\left(L_{\mathrm{mo}}\right)$
$2.0 \mathrm{~m}$

$-4.56 \mathrm{~m}$

\section{Surface roughness.}

Momentum roughness length $\left(z_{\mathfrak{n}}\right)$

$2 \mathrm{~mm}$

$\sigma_{u}\left(=\left\langle u^{\prime 2}\right\rangle^{1 / 2}\right)$ to the mean horizontal wind speed $\langle U\rangle$ is $1.20 / 2.44=0.49$ which is not very small (see Table I). However, we employ Taylor's hypothesis assuming that it is valid at least for inertial subrange scales. Therefore, the mean horizontal wind speed is used to convert time increments to space increments. ${ }^{45-48} \mathrm{We}$ also apply Taylor's hypothesis to the vertical velocity fluctuation so as to convert time to longitudinal distance increments; that is an observer moving in a frame of reference in the longitudinal direction at a speed $\langle U\rangle$ will note the measured time series vertical velocity fluctuations. In this case, the ratio $\sigma_{w}\left(=\left\langle w^{\prime 2}\right\rangle^{1 / 2}\right)$ to the mean horizontal wind speed $\langle U\rangle$ is 0.1 which is small. The resolvable wave number $K_{\mathrm{NY}}\left[=2 \pi /\left(\langle U\rangle\left(f_{s} / 2\right)^{-1}\right)\right]$ corresponding to the Nyquist frequency $\left(=f_{s} / 2\right)$ is $27.04 \mathrm{~m}^{-1}$, which is smaller than $42.2 \mathrm{~m}^{-1}$. Hence, wave-number distortions, discussed in Wyngaard, ${ }^{41}$ are not expected for these turbulence conditions and sampling frequency. For the temperature measurements, we note that the Prandtl number is about 0.7 and the inertial-convective range is considered rather than the inertial subrange.

\section{WAVELET STATISTICS}

In this section, relations between the Haar wavelet coefficients, the Fourier power spectrum, and the structure function are developed. We show how the orthonormal wavelet transform can be used to investigate intermittency effects on inertial subrange scaling.

\section{A. Relation between wavelet coefficients and Fourier power spectrum}

In Fourier analysis, the fundamental tool used to characterize turbulence is the power spectral density function $E(K)$. The function $E(K)$ represents the energy density contained in each wave-number band $d K$, and thus provides information regarding the importance of each scale of motion to the overall variance. However, important spatial informa- 
tion regarding location of events becomes implicit in the phase angle due to the global space-filling nature of Fourier transform. ${ }^{30}$ In this section, we relate the Haar wavelet coefficients to the Fourier power spectrum and show how spatial information can be expressed in an explicit manner using the wavelet coefficients.

The variance of the turbulence measurements, in terms of the wavelet coefficients, can be deduced from the conservation of energy in (11)

$$
\sigma^{2}=N^{-1} \sum_{m=1}^{m=M} \sum_{i=0}^{i=\left(2^{M-m}-1\right)}\left(W T^{(m)}[i]\right)^{2} .
$$

The total energy $T_{E}$ contained in scale $R_{m}\left(=2^{m} d y\right)$ can be computed from the sum of the squared wavelet coefficients at scale index $(m)$ using

$$
T_{E}=N^{-1} \sum_{i=0}^{i=\left(2^{M-m}-1\right)}\left(W T^{(m)}[i]\right)^{2},
$$

where $d y\left(=f_{s}^{-1}\langle U\rangle\right.$ from Taylor's hypothesis) is the measurement spacing in physical space. In order to compare the wavelet power spectrum to the Fourier power spectrum, we define a wave number $K_{m}$ corresponding to scale $R_{m}$ as

$$
K_{m}=2 \pi / R_{m}
$$

Hence, the power spectral density function $E\left(K_{m}\right)$ is computed by dividing $T_{E}$ by the change in wave number $\Delta K_{m}\left(=2 \pi 2^{-m} d y^{-1} \ln 2\right)$ so that

$$
E\left(K_{m}\right)=\frac{\left\langle\left(W T^{(m)}[i]\right)^{2}\right\rangle d y}{2 \pi \ln (2)},
$$

where $\langle\cdot\rangle$ is averaging in space over all values of $(i)$ for scale index $(m)$ (see Ref. 30). Hence, (15) demonstrates that the wavelet power spectrum at wave number $K_{m}$ is directly proportional to the average of the squared wavelet coefficients at that scale. Because the power at wave number $K_{m}$ is determined by averaging many squared wavelet coefficients, we expect the wavelet power spectrum to be smoother than its Fourier counterpart. This is apparent in Figs. 2(a) and 2(b) which display good agreement between Fourier and wavelet power spectra for velocity and temperature, respectively. The Fourier power spectrum was computed by square windowing 8192 points, cosine tapering $5 \%$ on each window edge, and averaging the resultant 4 power spectra $(N=32,768)$. The wavelet power spectrum was computed by (i) using the pyramidal algorithm defined by Eqs. (9) and (10) to obtain the wavelet coefficients over position index $(i)$ and scale index $(m)$, and (ii) using Eq. (15) in conjunction with computed wavelet coefficients from step (1) to obtain the wavelet power spectrum. Windowing is unnecessary for the wavelet power spectrum. An inertial subrange, whose signature is the $-5 / 3$ power law in the energy spectrum, was observed for three decades in the $U$ measurements [see Fig. 2(a)] and for about 1.5 decades in the $W$ and $T$ spectra [see Figs. 2(a) and 2(b)].
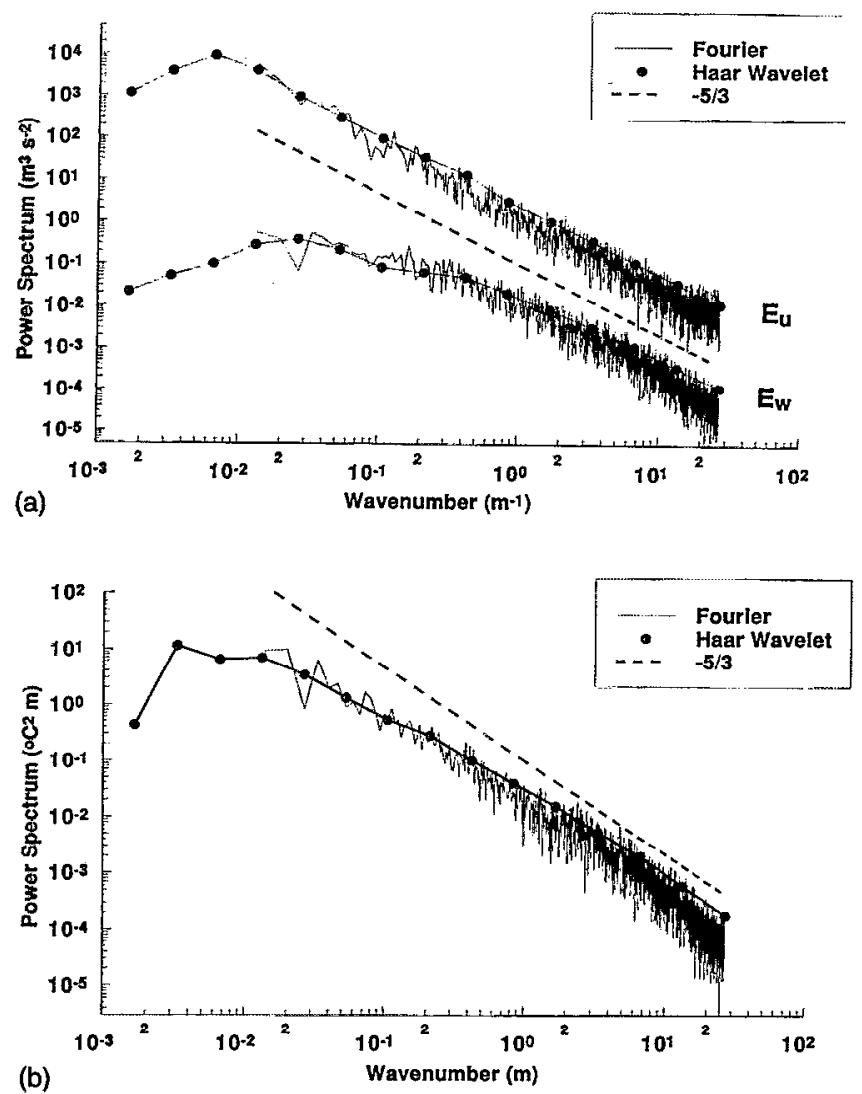

FIG. 2. (a) Comparison between the Fourier (solid line) and Haar wavelet (closed circle) power spectra for the longitudinal $(U)$ and vertical velocity $(W)$. The $U$ spectrum is shifted by two decades to permit comparison with $W$ spectrum at small wave number. Taylor's hypothesis is used to convert the time domain to wave-number domain. The $-5 / 3$ power law (dotted line) predicted by $\mathrm{K} 41$ is also shown. (b) Same as (a) but for temperature.

Since the wavelet power spectrum is directly proportional to the average of the squared wavelet coefficients, we can also determine the spatial standard deviation around that average using

$$
\begin{aligned}
S D_{E}\left(K_{m}\right)= & {[d y / 2 \pi \ln (2)]\left[\left\langle W T^{(m)}[i]^{4}\right\rangle\right.} \\
& \left.-\left\langle\left(W T^{(m)}[i]^{2}\right)\right\rangle^{2}\right]^{1 / 2} .
\end{aligned}
$$

A plot of $E\left(K_{m}\right)$ and $E\left(K_{m}\right)+S D_{E}$ gives a compact representation of the energy and its spatial variability at each scale, which is referred to as the "dual spectrum." 30,31 A better dimensionless indicator for the spatial energy variance is given by the coefficient of variation $C V_{E}$ defined as ${ }^{14,32}$

$$
C V_{E}\left(K_{m}\right)=S D_{E}\left(K_{m}\right) / E\left(K_{m}\right) \text {. }
$$

An example of the variation of $C V_{E}$ is shown in Fig. 3(a) for velocity and temperature. Notice in Fig. 3(a) that $C V_{E}$ increases as the wave number increases, indicating increased turbulent energy activity at smaller scales. The increased energy activity at smaller scales has classically been attributed to intermittency. ${ }^{49,50}$ A formal relation between $C V_{E}$ and the variance of the dissipation rate is given in Ref. 14 and will not be considered here. Hence, from a turbulent energy point of view, a key difference between wavelet and Fourier transforms is that Fourier transforms are nonlocal and therefore 

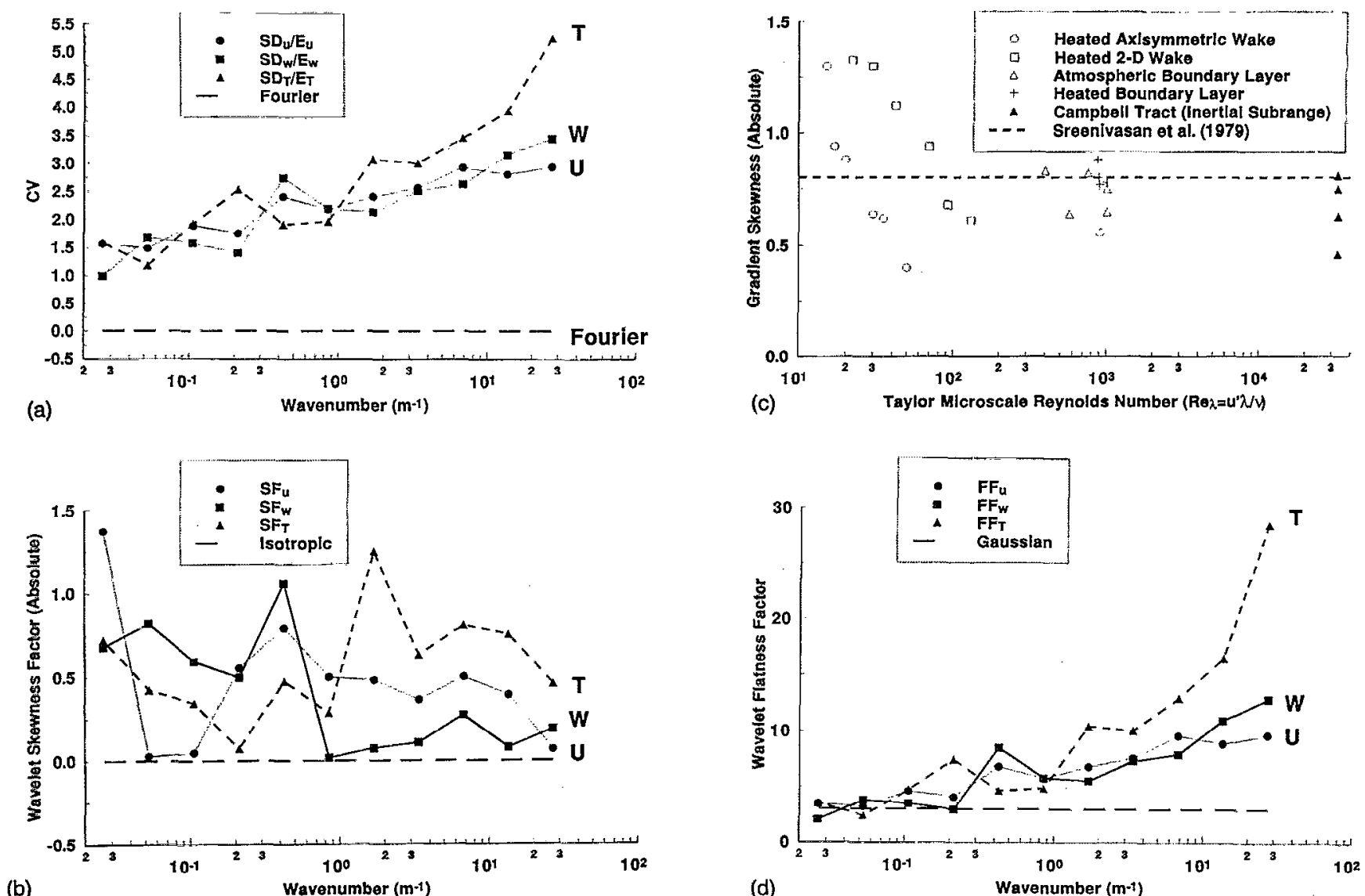

FIG. 3. (a) The cocfficient of variation (CV) as a function of wave number for longitudinal and vertical velocity, as well as temperature. The dotted line is the CV assumed by Fourier analysis. (b) The evolution of the magnitude of the wavelet skewness as a function of wave number for velocity and temperature. The dotted line is for locally isotropic turbulence. (c) Comparison between the wavelet predicted temperature gradient skewness and other temperature gradient skewness from experiments reported by Sreenivasan (1991). The solid triangles are the wavelet skewness for the inertial subrange. (d) Same as (b) but for the wavelet flatness factor. The dotted corresponds to a Gaussian distribution.

distribute the energy uniformly in space [i.e., $C V_{E}(K)=0$ ]. Another interesting point in Fig. 3(a) is that $C V_{E}$ for $T$ is much larger than $C V_{E}$ for $U$ or $W$. This may demonstrate that temperature is not simply advected by the velocity field, even at the small scales.

\section{B. Other wavelet statistical measures}

Two other useful statistical measures can be defined using the wavelet coefficients. The wavelet skewness $(S K)$ and the wavelet flatness factor $(F F)$ at scale index $(m)$ are defined as

$$
\begin{aligned}
& S F\left(K_{m}\right)=\left\langle\left(W T^{(m)}[i]\right)^{3}\right\rangle /\left\langle\left(W T^{(m)}[i]\right)^{2}\right\rangle^{3 / 2}, \\
& F F\left(K_{m}\right)=\left\langle\left(W T^{(m)}[i]\right)^{4}\right\rangle /\left\langle\left(W T^{(m)}[i]\right)^{2}\right\rangle^{4 / 2} .
\end{aligned}
$$

We note that the wavelet skewness and flatness factors in (18) and (19) can also be interpreted as the velocity and temperature horizontal gradients statistics if the following arguments are adopted.

(1) The differencing nature of the Haar wavelet transform, as can be noted from Eq. (9), results in direct proportionality between wavelet coefficients and velocity and temperature differences.
(2) The dimensionless ratios in Eqs. (18) and (19) are the same for differences and gradients, since the division by the wavelet width or separation distance required to convert differences to gradients in the numerator and the denominator cancel out. Thus, using these two arguments, $S K\left(K_{m}\right)$ and $F F\left(K_{m}\right)$ can be interpreted as gradient skewness and flatness factors at wave numbers $K_{m}$ (see, also, Refs. 14 and 22).

Figure 3(b) displays the magnitude of $S F(|S F|)$ as a function of wave number for the $U, W$, and $T$ measurements, respectively. For the $U$ measurements, $\left|S F_{u}\right|$ is about 0.4 within the inertial subrange and is consistent with the constant skewness hypothesis. ${ }^{2,4}$ A slightly higher value $\left(\left|S F_{\mu}\right|=0.5\right)$ was reported from numerical simulations by Kerr. ${ }^{51}$ We iterate again that these measurements invoke Taylor's hypothesis, but this may not affect our conclusions seriously since these wave numbers are large. We also compared our wavelet temperature skewness for inertial subrange scales with other temperature gradient measurements reported by Sreenivasan ${ }^{52}$ for the inertial subrange. These measurements are shown in Fig. 3(c). The dotted line in Fig. 3(c) is discussed in Ref. 52. The Taylor microscale $\lambda(=0.31 \mathrm{~m})$ in Fig. 3(c) was estimated from 


$$
\left.\lambda=\sigma_{u}\langle U\rangle /\left(\frac{\partial u^{\prime}}{\partial t}\right)^{2}\right)^{1 / 2}
$$

assuming locally isotropic turbulence. We note that large uncertainty is involved in the $\lambda$ estimate; however, for comparison with the reported temperature gradient data, ${ }^{52}$ this estimate is necessary. Figure 3(c) did not indicate a significant drop in the skewness as the Taylor microscale Reynolds number $\left(\sigma_{u} \lambda / \nu\right)$ increases. This agrees with the trend observed in Ref. 52 and conclusions.

The Haar wavelet $F F\left(K_{m}\right)$ measures the importance of the tails of the spatial probability distribution of the velocity and temperature horizontal gradients for wave number $K_{m}$. Figure 3(d) displays the variation of $F F\left(K_{m}\right)$ as a function of $K_{m}$. For all three turbulence measurements, the gradients at the smaller wave numbers appear to be nearly Gaussian. Non-Gaussian statistics in the velocity and temperature horizontal gradients exist within the inertial subrange. Again, notice how $F F$ is much larger for the temperature measurements when compared to the velocity measurements within the inertial subrange which is in agreement with the remarks of Fig. 3(a).

\section{Relation between wavelet coefficients and structure function}

As shown in (9) the Haar wavelet resembles a differencing operator, thus, it can be related to the $n$ th-order structure function for any flow variable $\phi(=U, W$, or $T)$ using

$$
\left\langle|\phi(x+r)-\phi(x)|^{n}\right\rangle \sim\left\langle\left|W T(i)^{(m)}\right|^{n}\right\rangle /\left(2^{m / 2} d y\right)^{n} .
$$

In (21), we applied the following: (1) the separation distance $r=2^{m} d y$; (2) the wavelet coefficients are proportional to $\phi(x$ $+r)-\phi(x)$ at position $x=\left(2^{m} i\right) d y$; (3) the amplitudes of the Haar wavelet coefficients are proportional to $\left(2^{m}\right)^{1 / 2}$; and (4) $\langle\cdot\rangle$ is the averaging operator of the wavelet coefficients over all values of the position index $(i)$ at scale index $(m)$. To study intermittency effects on (1), we modify the above relation and propose a conditional structure function to be discussed next.

\section{Conditional sampling and intermittency effects on K41}

In general, intermittency of turbulent fluids is symbolized by an on-off process so that at a certain time, the turbulent energy is only active in a certain fraction of the fluid volume. Hence, intermittency effects give rise to isolated large energetic events within an overall less energetic fluid volume. These events correspond to large squared wavelet coefficients at certain scale indices $(m)$ and position indices (i). Since the energy content is directly proportional to the square of the wavelet coefficients, we can classify the wavelet coefficients as either "active" (i.e., more energetic) or "passive." The distinction between active and passive must be based on some minimum energy threshold criterion. We chose this energy threshold to be related to the mean energy content at scale index $(m)$. This criterion can be formulated by comparing the squared wavelet coefficient at position $(i)$ and scale index $(m)\left[W T^{(m)}(i)^{2}\right]$ with the mean value
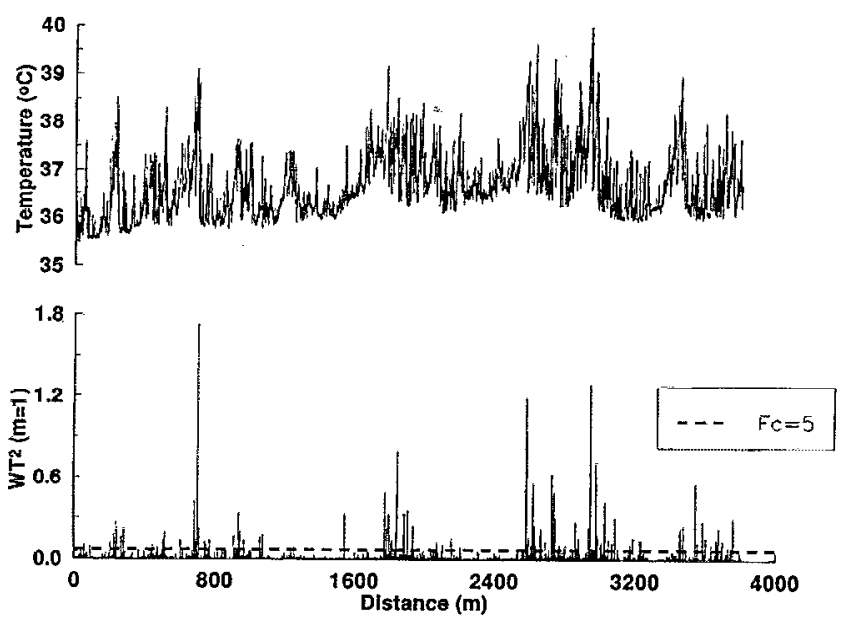

FIG. 4. The influence of sharp edges of main eddies on the squared wavelet coefficients for $m=1$. Taylor's hypothesis was used to convert time to space. The squared wavelet coefficients exceeding the dotted line $\left(F_{c}=5\right)$ are not considered in the conditional wavelet analysis at scale index $m=1$.

$\left\langle\left[W T^{(m)}(i)\right]^{2}\right\rangle$ at scale index $(m)$. In order to modify $(15)$ or (21) for intermittency investigation, we define an indicator function $I^{(m)}$ at scale index $(m)$ given by

$$
I^{(m)}=\left[\begin{array}{ll}
0 & \text { if }\left[W T^{(m)}(i)\right]^{2}>F_{c}\left\langle\left[W T^{(m)}(i)\right]^{2}\right\rangle \\
1 & \text { otherwise }
\end{array}\right],
$$

where $F_{c}$ is an arbitrary conditioning criteria that allows discrimination between the active and passive fluid volume. ${ }^{27-29}$ For cxample, if $F_{c}=5$, then all squared wavelet coefficients that are in excess of 5 times the average squared wavelet coefficient are set to zero for that scale index $(m)$. A relation between $F_{c}$, the mean dissipation rate, and the instantaneous dissipation rate is derived in Ref. 14 for locally isotropic turbulence.

An example of the application of the conditional wavelet criteria is shown in Fig. 4. Figure 4 shows the temperature measurements (the abscissa is converted into distance using Taylor's hypothesis) and the squared wavelet coefficients at scale index $m=1$. Notice that the large spikes in the squared wavelet coefficient (bottom plot) correspond to the sharp edges of the large-scale thermals in the temperature measurements. Therefore, the active wavelet coefficients result from large horizontal gradients in the temperature measurements. These large gradients are concentrated on the edges of these larger eddies (see Fig. 4, top plot), and hence, the large-scale motion directly influences the inertial subrange. The dotted line in Fig. 4 shows how the conditioning criteria eliminates the influence of these sharp edges from the wavelet transformed temperature measurements for scale index $m=1$. This conditioning criteria reduces the inertial subrange "contamination" produced by this large eddy motion.

We note here that Kuznetsov ${ }^{12}$ suggested that such interaction between the larger-scale eddy motion and inertial subrange scales is due to "external intermittency." It is not clear from their study whether such an intermittency is a genuine property of the Navier-Stokes equations or the result of 


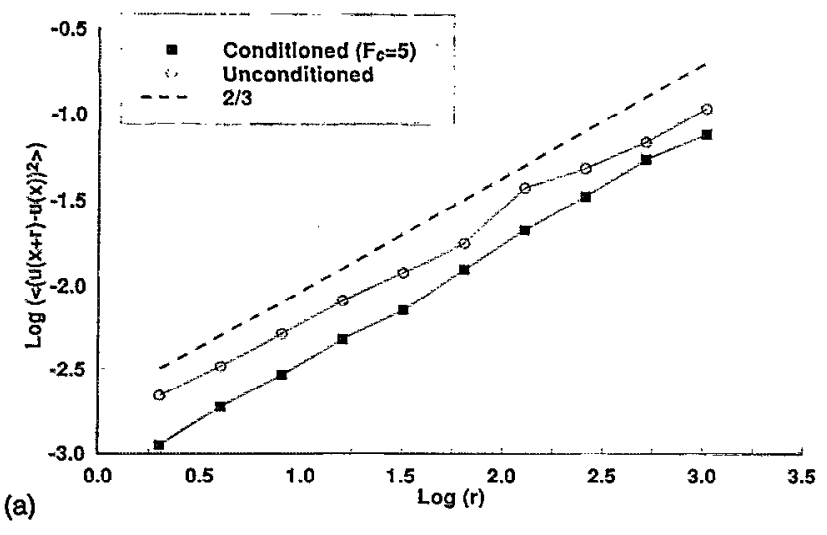

(a)

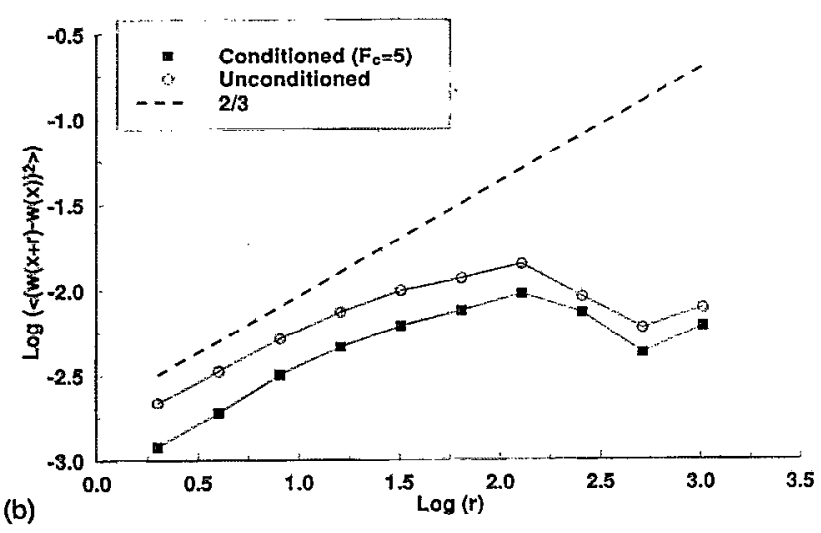

boundary conditions. For the purpose of this study, we do not focus on the origin of external intermittency but assume that the inertial subrange scaling as derived in $\mathrm{K} 41$ is contaminated by the large-scale motion.

Similar analysis was performed at all scales $(m)$ within the $-5 / 3$ power law. Hence, we can now consider a conditional power spectrum $E^{c}$ given by

$$
E^{c}\left(K_{m}\right)=\left\langle\left\langle\left(I^{(m)} W T^{(m)}[i]\right)^{2}\right\rangle\right\rangle d y / 2 \pi \ln (2),
$$

where $\langle\langle\cdot\rangle\rangle$ is now averaging in space over all nonzero values of $\left[I^{(m)} W T^{(m)}(i)\right]^{2}$. Also, $E^{c}$ represents the power spectrum of the less active fluid or the power spectrum in the absence of the contamination from larger eddies. We can also define the conditional $n$ th-order structure function ${ }^{14}$ by

$$
\begin{aligned}
& \left\langle|\phi(x+r)-\phi(x)|^{n}\right\rangle^{(c)} \\
& \quad \sim\left\langle\left\langle\left|I^{(m)} W T(i)^{(m)}\right|^{n}\right\rangle\right\rangle /\left(2^{m / 2} d y\right)^{n},
\end{aligned}
$$

where $\langle\langle\cdot\rangle\rangle$ is averaging over all nonzero values of $\left[I^{(m)} W T^{(m)}(i)\right]$. These conditional statistics can be computed by (i) using the pyramidal algorithm to calculate the Haar wavelet coefficients at each scale index $(m)$ and position index ( $i$ ); (ii) squaring these coefficients to obtain the energy content at cach scale index $(m)$ and position index $(i)$; (iii) averaging the squared wavelet coefficients for each scale index $(m)$; (iv) dividing the squared wavelet coefficient (at space index $i$ ) by the value computed in step (iii); (v) if this ratio is larger than some preset value for $F_{c}$, then set this coefficient to zero, otherwise leave as is; (vi) use Eq. (23) or Eq. (24) to determine the power spectrum or the $n$ th-order structure function with averaging performed over all nonzero

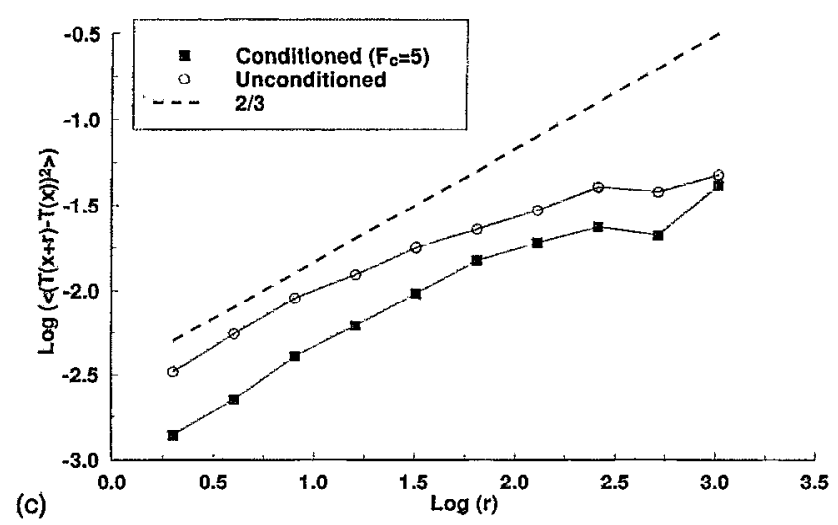

FIG. 5. (a) Comparison between conditioned and unconditioned longitudinal velocity second order structure function. The dotted line corresponds to K41 scaling. (b) Same as (a) but for vertical velocity. (c) Same as (a) but for tempcraturc.

values at scale index $(m)$. Repeat the above steps for all values of $(m)$ within the inertial subrange. The adequacy of this conditional sampling criteria for recovering $\mathrm{K} 41$ from the inertial subrange is discussed next using the velocity and temperature measurements.

\section{RESULTS AND DISCUSSION}

This section discusses the effects of intermittency on K41 for the velocity and temperature measurements using the conditional wavelet analysis for three cases: (1) $n=2$, (2) $n=3$, and (3) $n=6$. In each case, we check whether K41 is satisfactory for the velocity and temperature measurement when intermittency and other inertial subrange "contamination" are suppressed, and then we investigate the statistical structure of the events responsible for deviations from $\mathrm{K} 41$ scaling. We do not present theoretical details regarding intermittency models, but we focus more on the contrast between the conditioned (intermittency suppressed) and unconditioned statistics.

Case $1: n=2$. It is known that intermittency effects are generally small and may not be detectable for the structure function with $n=2$ (see Refs. 3 and 53). We test this hypothesis by comparing the unconditioned and conditioned $\left(F_{c}=5\right)$ structure functions of (24) for velocity and temperature, respectively. The results are presented in Figs. 5(a)5(c) for longitudinal and vertical velocities, and temperature, respectively. Both conditioned and unconditioned second order structure functions for velocity and temperature exhibit scaling laws that are in agreement with $K 41$ (slope $=2 / 3$ ). This supports the hypothesis that intermittency effects (ex- 
TABLE II. Summary of the regression statistics for the model $\log [\langle| \Delta u, w$, or $\left.\left.\left.T\right|^{n}\right\rangle\right]=A \log [r]+B$. Mixed moments of the form $\log \left[\left\langle\Delta T^{2} \Delta u\right\rangle\right.$, $\left.\left\langle\Delta T^{4} \Delta u^{2}\right\rangle\right]=A \log [r]+B$ are also presented for intermittency parameter determination. The coefficient of determination $\left(R^{2}\right)$ and the standard error of estimate (SEE) are shown. The conditioned statistics are for the conditioning criterion $F_{c}=5$. Here, $(C)$ are conditioned structure functions, and $(U)$ are unconditioned structure functions. The number of points used in the regression analysis is also shown.

\begin{tabular}{|c|c|c|c|c|c|c|c|}
\hline Variable & $n$ & $\begin{array}{l}\text { Slope } \\
\text { (A) }\end{array}$ & $\begin{array}{l}\text { Intercept } \\
\text { (B) }\end{array}$ & $R^{2}$ & SEE & $\begin{array}{l}\text { Number of } \\
\text { points }\end{array}$ & $(C) /(U)$ \\
\hline$u$ & 2 & $\begin{array}{l}0.68 \\
0.64\end{array}$ & $\begin{array}{l}-3.163 \\
-2.875\end{array}$ & $\begin{array}{l}0.999 \\
0.994\end{array}$ & $\begin{array}{l}0.0159 \\
0.0401\end{array}$ & $\begin{array}{l}10 \\
10\end{array}$ & $\begin{array}{l}C \\
U\end{array}$ \\
\hline$w$ & 2 & $\begin{array}{l}0.67 \\
0.60\end{array}$ & $\begin{array}{l}-3.120 \\
-2.837\end{array}$ & $\begin{array}{l}0.996 \\
0.997\end{array}$ & $\begin{array}{l}0.0189 \\
0.0135\end{array}$ & $\begin{array}{l}4 \\
4\end{array}$ & $\begin{array}{l}C \\
U\end{array}$ \\
\hline$T$ & 2 & $\begin{array}{l}0.69 \\
0.56\end{array}$ & $\begin{array}{l}-3.050 \\
-2.606\end{array}$ & $\begin{array}{l}0.997 \\
0.983\end{array}$ & $\begin{array}{l}0.0245 \\
0.0455\end{array}$ & $\begin{array}{l}6 \\
6\end{array}$ & $\begin{array}{l}C \\
U\end{array}$ \\
\hline$u$ & 3 & $\begin{array}{l}1.02 \\
0.89\end{array}$ & $\begin{array}{l}-4.476 \\
-3.836\end{array}$ & $\begin{array}{l}0.999 \\
0.992\end{array}$ & $\begin{array}{l}0.0323 \\
0.0750\end{array}$ & $\begin{array}{l}10 \\
10\end{array}$ & $\begin{array}{l}C \\
U\end{array}$ \\
\hline$w$ & 3 & $\begin{array}{l}0.98 \\
0.79\end{array}$ & $\begin{array}{l}-4.421 \\
-3.780\end{array}$ & $\begin{array}{l}0.999 \\
0.999\end{array}$ & $\begin{array}{l}0.0254 \\
0.0129\end{array}$ & $\begin{array}{l}4 \\
4\end{array}$ & $\begin{array}{l}C \\
U\end{array}$ \\
\hline$T$ & 3 & $\begin{array}{l}0.99 \\
0.66\end{array}$ & $\begin{array}{l}-4.255 \\
-3.252\end{array}$ & $\begin{array}{l}0.995 \\
0.964\end{array}$ & $\begin{array}{l}0.0466 \\
0.0801\end{array}$ & $\begin{array}{l}6 \\
6\end{array}$ & $\begin{array}{l}C \\
U\end{array}$ \\
\hline$u$ & 6 & $\begin{array}{l}1.99 \\
1.50\end{array}$ & $\begin{array}{l}-8.148 \\
-5.905\end{array}$ & $\begin{array}{l}0.999 \\
0.971\end{array}$ & $\begin{array}{l}0.0706 \\
0.0247\end{array}$ & $\begin{array}{l}10 \\
10\end{array}$ & $\begin{array}{l}C \\
U\end{array}$ \\
\hline$w$ & 6 & $\begin{array}{l}1.88 \\
0.88\end{array}$ & $\begin{array}{l}-8.00 \\
-5.33\end{array}$ & $\begin{array}{l}0.997 \\
0.943\end{array}$ & $\begin{array}{l}0.0456 \\
0.0920\end{array}$ & $\begin{array}{l}4 \\
4\end{array}$ & $\begin{array}{l}C \\
U\end{array}$ \\
\hline$T$ & 6 & $\begin{array}{c}1.97 \\
0.921\end{array}$ & $\begin{array}{l}-7.602 \\
-4.339\end{array}$ & $\begin{array}{l}0.992 \\
0.916\end{array}$ & $\begin{array}{l}0.095 \\
0.154\end{array}$ & $\begin{array}{l}5 \\
5\end{array}$ & $\begin{array}{l}C \\
U\end{array}$ \\
\hline$\Delta T^{2} \Delta u$ & $* *$ & $\begin{array}{l}0.95 \\
1.38\end{array}$ & $\begin{array}{l}-1.82 \\
-2.09\end{array}$ & $\begin{array}{l}0.990 \\
0.896\end{array}$ & $\begin{array}{l}0.06 \\
0.16\end{array}$ & $\begin{array}{l}8 \\
8\end{array}$ & $\begin{array}{l}C \\
U\end{array}$ \\
\hline$\Delta T^{4} \Delta u^{2}$ & $* *$ & $\begin{array}{l}1.76 \\
1.54\end{array}$ & $\begin{array}{l}-2.61 \\
-0.57\end{array}$ & $\begin{array}{l}0.993 \\
0.943\end{array}$ & $\begin{array}{l}0.11 \\
0.21\end{array}$ & $\begin{array}{l}8 \\
8\end{array}$ & $\begin{array}{l}C \\
U\end{array}$ \\
\hline
\end{tabular}

ternal or internal) may not be very significant for $n=2$ (see Refs. 54 and 55 for a possible physical explanation). We also present a summary of the regression statistics for the regression model $\log \left[D^{2}(r)\right]=A \log [r]+B$ in Table II. Notice in Table II that the coefficient of determination $\left(R^{2}\right)$ for the regression model is in excess of 0.99 ; hence, the determination of scaling laws from wavelet structure functions appears to be very reliable. Also, in order to check the effects of our conditioning criteria $\left(F_{c}\right)$ on the slope of the structure function, we performed the same analysis for $F_{c}=4,5,7$, and 10. The slope variation $A$ (for $n=2$ ) did not differ by more than 0.008 . Some limitations of the proposed conditional sampling scheme are discussed in Ref. 14.

Case 2: $n=3$. In Landau and Lifshitz, ${ }^{4}$ a relation between the third-order structure function and $(r)$ is given by

$$
\left\langle\left|(\Delta u)^{3}\right|\right\rangle=\frac{4}{5}\langle\epsilon\rangle r .
$$

The above relation was derived from the Navier-Stokes equations, and thus is independent of any assumptions implicit in K41 or any intermittency corrections to K41. For the temperature measurements, an equivalent formulation to (25) is given by

$$
\left\langle\left|(\Delta T)^{2} \Delta u\right|\right\rangle=\frac{4}{3}\left\langle N_{T}\right\rangle r
$$

where $N_{T}$ is temperature dissipation rate (see Ref. 2, p. 400 , for derivation).

If the intermittency is "internal" in the sense of Kolmogorov, ${ }^{8}$ then our conditioned and unconditioned statistics should both reproduce the $r^{1}$ dependence. Using (21) and (25), with $F_{c}=5$ and $n=3$, the unconditioned and conditioned third-order structure functions are compared in Figs. 6(a) and $6(\mathrm{~b})$, for the longitudinal and vertical velocities, and the mixed second-moment temperature and first-moment velocity differences in Fig. 6(c), respectively. Notice in Figs. $6\left(\right.$ a) $-6(c)$ that an $r^{1}$ power law was not observed for the unconditioned structure function. This indicates that the intermittency and other sources of inertial subrange contamination are responsible for deviations from K41 as suggested by Kuznetsov. ${ }^{12}$ The conditioned slopes (see Table II) are in good agreement with Landau and Lifshitz ${ }^{4}$ predictions (and are consistent with $\mathrm{K} 41$ ). This analysis demonstrates the usefulness of the third-order structure function to identify possible contamination of the inertial subrange scaling for locally isotropic turbulence.

Case 3: $n=6$. The sixth-order structure function can be related to the dissipation correlation function from

$$
\frac{\left\langle(\Delta u)^{6}\right\rangle}{r^{2}} \sim\langle\epsilon(x) \epsilon(x+r)\rangle,
$$

where the dissipation correlation function is given by

$$
\langle\epsilon(x) \epsilon(x+r)\rangle \sim\left(\frac{L}{r}\right)^{\mu}
$$

and $\mu$ is the intermitlency parameter. ${ }^{3,7,8,56}$ The value of $\mu$ has been the subject of extensive research and its value appears to vary between 0.15 and 0.5 . Kuznetsov ${ }^{12}$ showed that the large variation in $\mu$ is due to external intermittency. From (27) and (28), we see that the sixth-order structure function is related to $\mu$ using

$$
\left\langle(\Delta u)^{6}\right\rangle \sim r^{2-\mu} .
$$

For the temperature measurements, an equivalent formulation to (29) is given by

$$
\left\langle\left|(\Delta T)^{4} \Delta u^{2}\right|\right\rangle \sim r^{2-\mu_{T}},
$$

where $\mu_{T}$ is the thermal intermittency parameter due to the dissipation correlation function given by

$$
\left\langle N_{T}(x) N_{T}(x+r)\right\rangle \sim\left(\frac{L_{T}}{r}\right)^{\mu_{T}}
$$

where $L_{T}$ is the temperature integral length scale. ${ }^{57}$

We now evaluate the performance of the conditional wavelet analysis for reproducing K41 scaling for $n=6$ and suppressing intermittency buildup $\left(\mu=0, \mu_{T}=0\right)$ for both velocity and temperature. Using (21) and (24) with $F_{c}=5$ and $n=6$, we compare the unconditioned and conditioned sixth-order wavelet velocity structure function in Figs. 7(a) and $7(\mathrm{~b})$, and the mixed velocity/temperature structure function in Fig. 7(c), respectively. The slope of conditioned sixthorder structure function is $2.0(=2-\mu)$ indicating that intermittency is well suppressed $(\mu=0)$ for the higher-order statistics in both velocity components. Recall that the conditioning criteria is based on second-order statistics (wavelet 


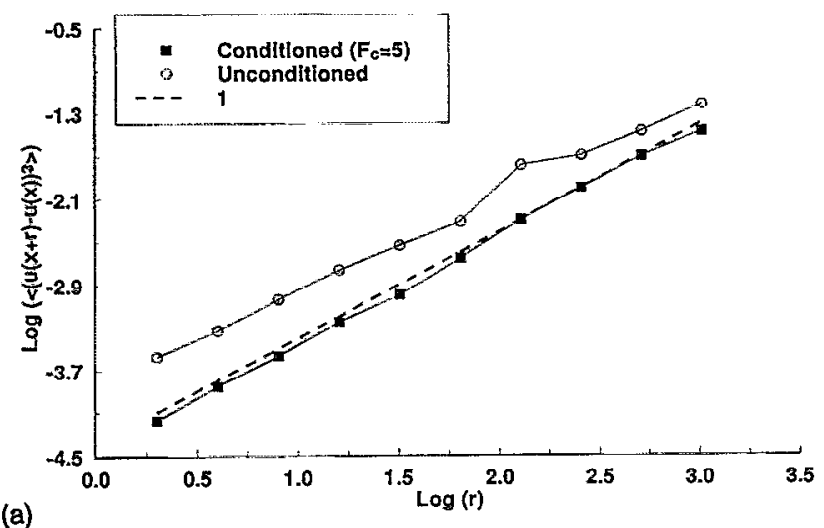

(a)

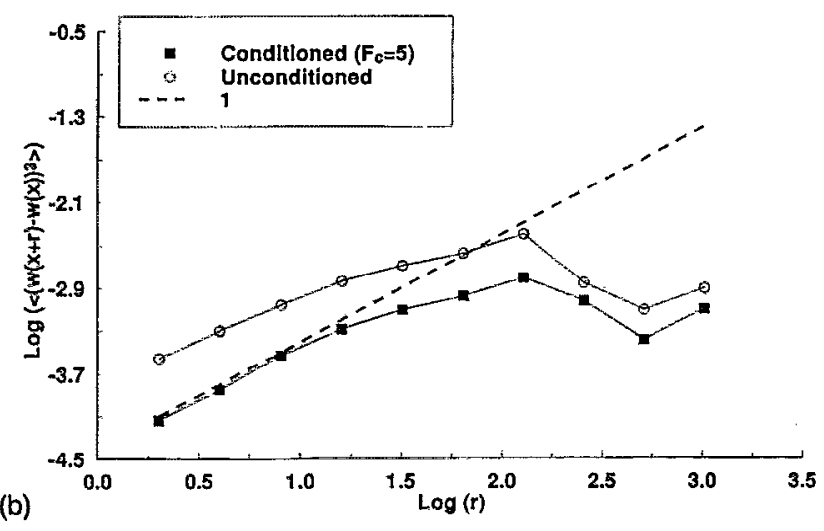

power spectrum), yet intermittency was suppressed in the sixth-order statistics. For the temperature measurements, it is clear that $\mu_{T}(=0.46)$, determined from the unconditioned mixed moments statistics of Table II, is larger than other
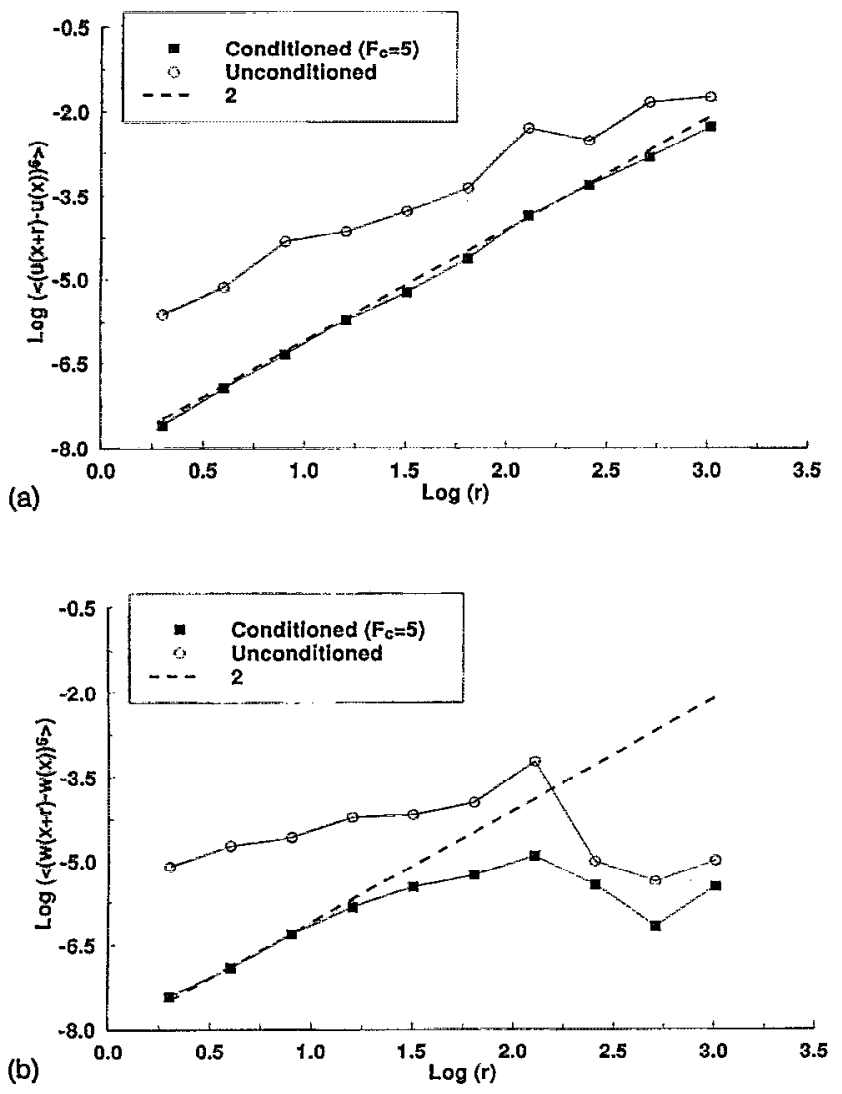

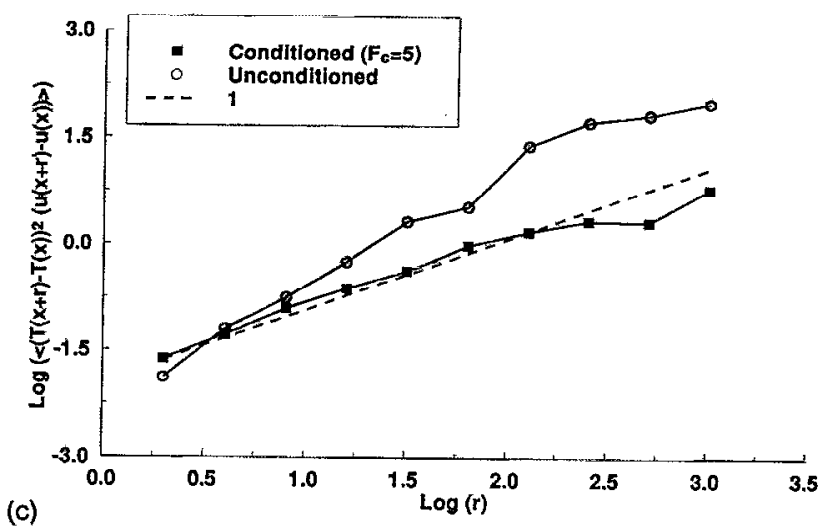

FIG. 6. (a) Same as Fig. 5(a) but for third-order structure function. (b) Same as Fig. 5(a) but for vertical velocity and third-order structure function. (c) Same as Fig. 5(a) but for the mixed second-order temperature and first-order velocity structure function.

laboratory reported values $(0.2-0.25)$ indicating again that external contamination of the inertial subrange significantly contributes to deviations from K41. However, the conditioned statistics did not give $\mu_{T}=0$ but rather a value of 0.24

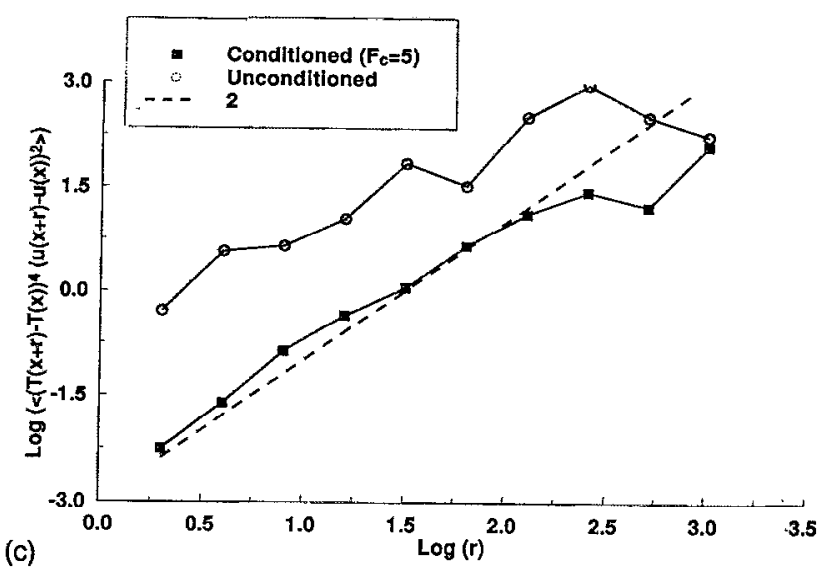

FIG. 7. (a) Same as Fig. 5(a) but for sixth-order structure function. (b) Same as Fig. 5(a) but for vertical velocity and sixth-order structure function. (c) Same as Fig. 5(a) but for the mixed fourth-order temperature and secondorder velocity structure function. 


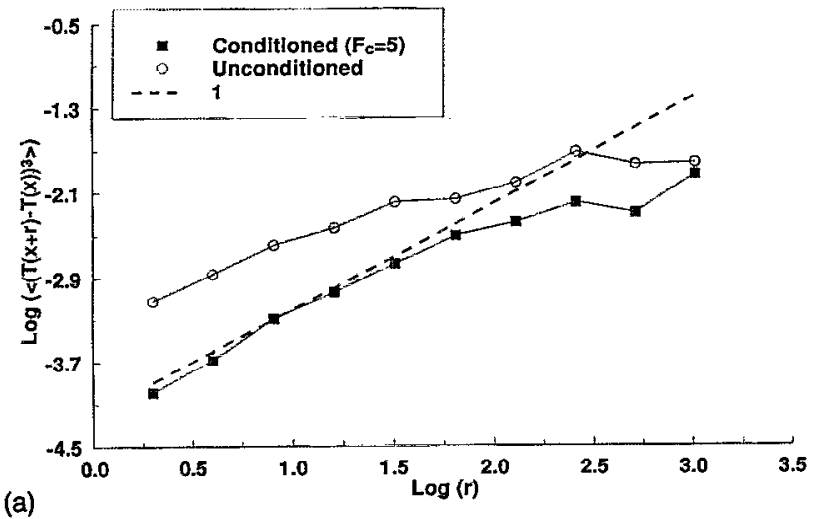

(a)

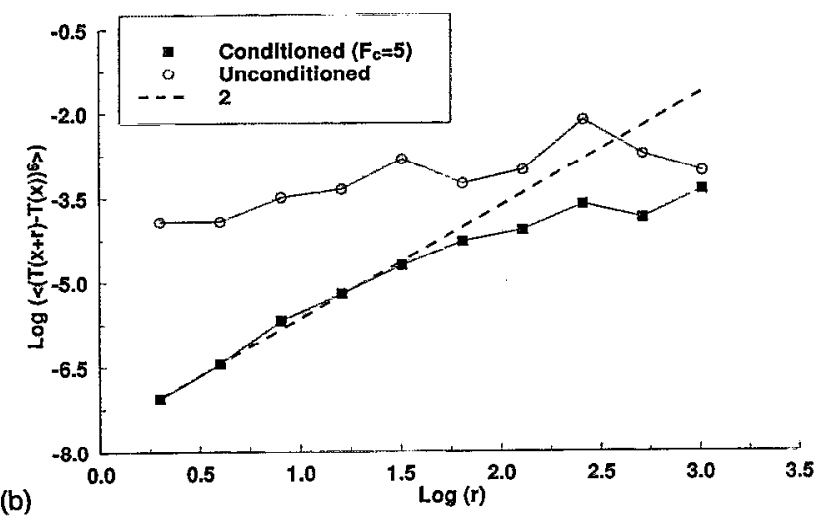

FIG. 8. (a) Same as Fig. 5(a) but for third order and temperature. (b) Same as Fig. 5(a) but for sixth order and temperature.

which is identical to that reported in Ref. 57 for ASL measurements.

\section{A. Further comments on extending $\mathrm{K} 41$ to temperature}

Analogous to (1), the $n$ th-order temperature structure function is given by

$$
\left\langle[\Delta T]^{n}\right\rangle=\left\langle[T(x+r)-T(x)]^{n}\right\rangle=C_{n}^{(T)}\left\langle N_{T}^{n / 2} \epsilon^{-n / 6}\right\rangle r^{n / 3}
$$

within the inertial subrange. ${ }^{58}$ We investigate whether (32) is recovered using the proposed conditional wavelet analysis. Figures 8(a) and 8(b) present these results for $n=3$ and $n=6$ (using $F_{c}=5$ ), respectively. Notice that the scaling in (32) is recovered by the conditional wavelet analysis and all inertial subrange contamination is eliminated (see Table I).

\section{B. K41 and non-Gaussian statistics}

Kraichnan ${ }^{6}$ suggested that $\mathrm{K} 41$ is consistent with the concept of an inertial cascade if the velocity statistics within the inertial subrange do not differ significantly from Gaussian. We therefore consider the structure of the velocity statistics and its relation to $\mathrm{K} 41$ scaling by noting that conditional wavelet analysis recovers $\mathrm{K} 41$ and eliminates both internal and external intermittency effects. The relation between Gaussian behavior and conditioned velocity and temperature statistics within the inertial subrange can be achieved by computing the conditioned and unconditioned

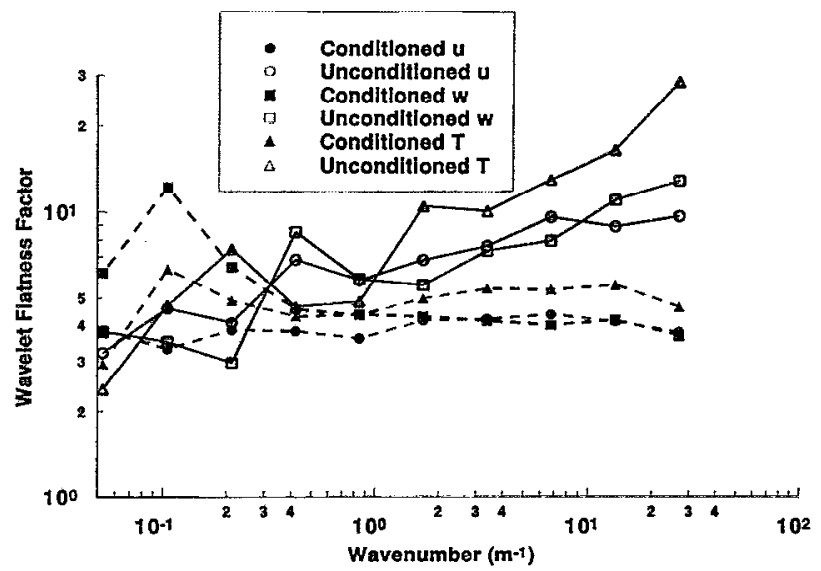

FIG. 9. Comparison between conditioned and unconditioned wavelet flatness factor for velocity and temperature. The closed symbols are conditioned and the open symbols are unconditioned.

wavelet flatness factor for the inertial subrange scales. ${ }^{14}$ The results are summarized in Fig. 9. Notice that the conditioned $F F\left(R_{m}\right)$ are nearly Gaussian (i.e., $F F \sim 3$ ) for all inertial subrange scales (velocity and temperature) while the unconditioned wavelet statistics are non-Gaussian. This analysis clearly indicates that $\mathrm{K} 41$ (which was obtained using the conditional wavelet analysis) is associated with nearGaussian statistics in agreement with the arguments of Ref. 6.

\section{CONCLUSIONS}

Triaxial sonic anemometer velocity and temperature measurements at $2.0 \mathrm{~m}$ above a uniform bare soil surface were used to investigate intermittency buildup in the inertial subrange. The power spectrum of the longitudinal velocity measurements exhibits a $-5 / 3$ power law for three decades allowing detailed investigation of scaling laws in the inertial subrange. The temperature and vertical velocity exhibit a $-5 / 3$ slope for about 1.5 decades. In order to describe spacescale relations in the inertial subrange, we utilized orthonormal wavelets. The orthonormal wavelet representation is well suited for this investigation, since the basis function is orthogonal and mutual independence of the wavelet expansion coefficients is guaranteed. In addition, it was shown that the expansion coefficients can be related directly to quantities commonly used in conventional turbulence analysis. Relations between the Haar wavelet coefficients and the Fourier power spectrum, as well as relations with the $n$ th-order structure function were derived. A comparison between Fourier and wavelet power spectra was also carried out. Good agreement between the two spectra was noted for both velocity and temperature measurements. Furthermore, we demonstrated the usefulness of orthonormal wavelet transforms in characterizing the spatial variation of turbulent energy at different scales. Since intermittency buildup in the inertial subrange is related to local energy containing events, a conditional wavelet scheme was developed. The conditional wavelet scheme efficiently suppressed intermittency and 
other external contamination within the inertial subrange. K41 statistics, up to sixth order, were obtained when intermittency was suppressed from the wavelet coefficients. The recovery of $\mathrm{K} 41$ up to sixth-order statistics from limited number of measurements also demonstrates the robustness of orthonormal wavelet transforms. It was found that the sharp edges of the large eddies directly contribute to inertial subrange scales. It was also found that intermittency did not significantly affect second-order statistics. We demonstrated that intermittency as well as other external effects are directly responsible for non-Gaussian statistics in velocity and temperature horizontal gradients in the inertial subrange, while K41 appears to be consistent with near-Gaussian statistics for these gradients.

\section{ACKNOWLEDGMENTS}

The authors would like to thank John Albertson for his assistance and Mike Mata for his help in maintaining the field site.

We are grateful for the funding support from the National Science Foundation (NSF) under Grant No. EAR-9304331, U.S. Geological Survey (USGS), Water Resources Center (WRC) under Grant No. W-812, Kearney Foundation, and UC Davis superfund Grant No. 5 P42ES04699-07.

${ }^{1}$ A. N. Kolmogorov, "The local structure of turbulence in incompressible viscous fluid for very large Reynolds numbers," Dokl. Akad. Nauk SSSR 4, 299 (1941).

${ }^{2}$ A. S. Monin and A. M. Yaglom, Statistical Fluid Mechanics Vol. II, edited by J. Lumley (MIT Press, Cambridge, MA, 1975).

${ }^{3}$ F. Anselmet, Y. Gagne, E. J. Hopfinger, and R. A. Antonia, "High-order velocity structure functions in turbulent shear flows," J. Fluid Mech. 140, 63 (1984).

${ }^{4}$ L. D. Landau and E. M. Lifshitz, Fluid Mechanics (Pergamon, New York, 1986), $539 \mathrm{pp}$.

${ }^{5}$ U. Frisch, "From global scaling à la Kolmogorov to local multifractal scaling in fully developed turbulence," in Turbulence and Stochastic Processes: Kolmogorov's Ideas 50 Years On, edited by J. C. R. Hunt, M. O. Phillips, and D. Williams (Royal Society, London, 1991), 240 pp.

${ }^{\circ} \mathrm{R}$. Kraichnan, "Turbulent cascade and intermittency growth," Turbulence and Stochastic Processes: Kolmogorov's Ideas 50 Years On, edited by J. C. R. Hunt, M. O. Phillips, and D. Williams (Royal Society, London, 1991), $240 \mathrm{pp}$.

${ }^{7}$ U. Frisch, P. L. Sulem, and M. Nelkin, "A simple dynamical model of intermittent fully developed turbulence," J. Fluid Mech. 87, 719 (1978).

${ }^{8} \mathrm{~A}$. N. Kolmogorov, "A refinement of previous hypotheses concerning the local structure of turbulence in a viscous incompressible fluid at high Reynolds number," J. Fluid Mech. 13, 82 (1962).

${ }^{9} \mathrm{C}$. Meneveau and K. R. Sreenivasan, "Simple multifractal cascade model for fully developed turbulence," Phys. Rev. Lett. 59, 1427 (1987).

${ }^{10} \mathrm{C}$. Meneveau and $\mathrm{K}$. R. Sreenivasan, The multifractal nature of turbulent cnergy dissipation," J. Fluid Mech. 224, 429 (1991).

${ }^{11} \mathrm{E}$. Aurell, U. Frisch, J. Lutsko, and M. Vergassola, "On the multifractal properties of the energy dissipation derived from turbulence data," J. Fluid Mech. 238, 467 (1992).

${ }^{12}$ V. R. Kuznetsov, A. A. Praskovsky, and V. A. Sabelnikov, "Fine-scale turbulence structure of intermittent shear flows," J. Fluid Mech. 243, 595 (1992).

${ }^{13}$ J. C. Kaimal, J. C. Wyngaard, Y. Izumi, and O. R. Cote, "Spectral characteristics of surface layer turbulence," Q. J. R. Metcorol. Soc. 98, 563 (1972).

${ }^{14}$ G. G. Katul, J. D. Albertson, C. C. Chu, and M. B. Parlange, "Intermittency in atmospheric surface layer turbulence: The orthonormal wavelet representation," in Wavelets in Geophysics (in press, 1994).

${ }^{15} \mathrm{~J}$. Lumley and $\mathrm{H}$. Panofsky, The structure of Atmospheric Turbulence (Wiley, New York, 1964), $237 \mathrm{pp}$.

${ }^{16}$ E. Barcy, A. Arneodo, U. Frisch, Y. Gagne, and E. Hopfinger, "Wavelet analysis of fully developed turbulence data and measurement of scaling exponents," in Turbulence and Coherent Structures, edited by O. Metais, and M. Lesieur (Kluwer Academic, Dordrecht, The Netherlands, 1990), $450 \mathrm{pp}$.

${ }^{17}$ R. Everson, L. Sirovich, and K. R. Sreenivasan, "Wavelet analysis of the turbulent jet," Phys. Lett. A 145, 314 (1990).

${ }^{18}$ F. Argoul, A. Arneodo, G. Grasseau, Y. Gagne, E. J. Hopfinger, and U. Frisch, "Wavelet analysis of turbulence reveals the multifractal nature of the Richardson cascade," Nature 338, 51 (1989).

${ }^{19} \mathrm{R}$. Benzi and M. Vergassola, "Optimal wavelet analysis and its application to two dimensional turbulence," Fluid Dyn. Res. 8, 117 (1991).

${ }^{20} \mathrm{M}$. Vergassula and U. Frisch, "Wavelet transforms of self similar processes," Physica D 54, 58 (1991).

${ }^{21} \mathrm{~J}$. Liandrat and F. Moret-Bailly, "The wavelet transform: some applications to fluid dynamics and turbulence," Eur. J. Mech. B/Fluids 9, 1 (1991).

${ }^{22} \mathrm{~L}$. Mahrt, "Eddy asymmetry in the shear heated boundary layer," J. Atmos. Sci. 48, 472 (1991).

${ }^{23} \mathrm{~S}$. Collineau and Y. Brunet, "Detection of turbulent coherent motions in a forest canopy. Part I: Wavelet analysis," Boundary-Layer Meteorol. 65, 357 (1993).

${ }^{24}$ Y. Meyer, "Orthonormal wavelets," in Wavelets: Time-Frequency Methods and Phase Space, edited by J. M. Combcs, A. Grossmann, Ph. Tchamitchian, (Springer-Verlag, Berlin, 1989), 315 pp.

${ }^{25}$ I. Daubechies, Ten Lectures on Wavelets, CBMS-NSF Regional Conference Series in Applied Mathematics (SIAM, Philadelphia, PA, 1992).

${ }^{26}$ C. K. Chui, An Introduction to Wavelets (Academic, New York, 1992), 264 pp.

${ }^{27}$ M. Yamada and K. Ohkitani, “Orthonormal expansion and its application to turbulence," Prog. Theor. Phys.: Progress Lett. 86, 819 (1990).

${ }^{28} \mathrm{M}$. Yamada and $\mathrm{K}$. Ohkitani, "Orthonormal wavelet analysis of turbulence," Fluid Dyn. Res. 8, 101 (1991).

${ }^{29} \mathrm{M}$. Yamada and K. Ohkitani, "An identification of energy cascade in turbulence by orthonormal wavelet analysis," Prog. Theor. Phys. 86, 799 (1991).

${ }^{30} \mathrm{C}$. Meneveau, "Analysis of turbulence in the orthonormal wavelet representation," J. Fluid Mech. 232, 469 (1991).

${ }^{31} \mathrm{C}$. Meneveau, "Dual spectra and mixed energy cascade of turbulence in the wavelet representation," Phys. Rev. Lett. 11, 1450 (1991).

${ }^{32} \mathrm{G}$. G. Katul and M. B. Parlange, "On the active role of temperature in surface layer turbulence," J. Atmos. Sci. (in press).

${ }^{33} \mathrm{~A}$. Grossmann, R. Kronland-Martinet, and J. Morlet, "Reading and understanding continuous wavelet transforms," in Wavelets: Time-Frequency Methods and Phase Space, edited by J. M. Combes, A. Grossmann, and $\mathrm{Ph}$. Tchamitchian (Springer-Verlag, Berlin, 1989), 315 pp.

${ }^{34} \mathrm{G}$. David, Wavelets and Singular Integrals on Curves and Surfaces (Springer-Verlag, Berlin, 1992), $109 \mathrm{pp}$.

${ }^{35}$ I. Daubechies, "Orthonormal bases of compactly supported wavelets," Comm. Pure Appl. Math. XLI, 909 (1988).

${ }^{30} \mathrm{M}$. Farge, "Wavelet transforms and their applications to turbulence," Annu. Rev. Fluid Mech. 24, 395 (1992).

${ }^{37} \mathrm{~S}$. Mallat, "A theory for multiresolution signal decomposition: The wavelet representation," IEEE Trans. Pattern Analy. Mach. Intelligence 11, 674 (1989).

${ }^{38} \mathrm{~S}$. Mallat, "Multiresolution approximations and wavelet orthonormal bases of $L^{2}(R)$," Trans. Am. Math. Soc. 315, 69 (1989).

${ }^{39}$ G. Beylkin, R. Coifman, and V. Rokhlin, "Fast wavelet transforms and numerical algorithms. I.," Comm. Pure Appl. Math. XLIV, 141 (1991).

${ }^{40} \mathrm{G}$. Beylkin, R. Coifman, and V. Rokhlin, "Wavelets in numerical analysis," in Wavelets and their Applications, edited by M. B. Ruskai, G. Beylkin, R. Coifman, I. Daubechies, S. Mallat, Y. Meyer, and L. Raphael (Jones and Bartlett, Boston, 1991), 474 pp.

${ }^{41} \mathrm{~J}$. C. Wyngaard, "Cup, propeller, vane, and sonic anemometer in turbulence research," Annu. Rev. Fluid Mech. 13, 399 (1981).

${ }^{42} \mathrm{C}$. A. Friehe, "Fine-scale measurements of velocity, temperature, and humidity in the atmospheric surface layer," in Probing the Atmospheric Boundary Layer, edited by D. Lenschow (American Meteorological Society, Washington, DC, 1986), $269 \mathrm{pp}$.

${ }^{43}$ V. Suomi and J. A. Businger, "Principle of the sonic anemometerthermometer," Geophys. Res. Papers, 59, (1959).

${ }^{44} \mathrm{~B}$. Tanner, "Use requirements for Bowen Ratio and eddy-correlation determination of evapotranspiration," in Proceedings of the 1988 Specialty Conference of the Irrigation and Drainage Division, American Society of Civil Engineers, 19-21 July, 1988. 
${ }^{45} \mathrm{~J}$. Lumley, "Interpretation of time spectra measured in high intensity shear flows," Phys. Fluids 6, 1056 (1965).

${ }^{46} \mathrm{D}$. Powell and C. E. Elderkin, "An investigation of the application of Taylor's hypothesis to atmospheric boundary layer turbulence," J. Atmos. Sci. 31, 990 (1974).

${ }^{47} \mathrm{~J}$. C. Wyngaard and S. F. Clifford, "Taylor's hypothesis and highfrequency turbulence spectra," J. Atmos. Sci. 34, 922 (1977).

${ }^{48} \mathrm{R}$. Stull, An Introduction to Boundary Layer Meteorology (KluwerAcademic, Dordrecht, The Netherlands, 1988), $666 \mathrm{pp}$.

${ }^{49}$ W. D. McComb, The Physics of Fluid Turbulence (Oxford Science, London, 1992), 572 pp.

${ }^{50} \mathrm{M}$. Lesieur, Turbulence in Fluids (Kluwer-Academic, Dordrecht, The Netherlands, 1987), 286 pp.

${ }^{51}$ R. M. Kerr, "Velocity, scalar and transfer spectra in numerical turbulence," J. Fluid Mech. 211, 309 (1990).

${ }^{52} \mathrm{~K}$. R. Sreenivasan, "On local isotropy of passive scalars in turbulent shear flows," in Turbulence and Stochastic Processes: Kolmogorov's Ideas 50
Years On, edited by J. C. R. Hunt, M. O. Phillips, and D. Williams (Royal Society, London, 1991), $240 \mathrm{pp}$.

${ }^{53} \mathrm{~V}$. Yakhot, Z. S. She, and S. A. Orszag, "Deviations from the classical Kolmogorov theory of the inertial range of homogeneous turbulence," Phys. Fluids A 1, 289 (1989).

${ }^{54} \mathrm{~A}$. J. Chorin, "Spectrum, dimension, and polymer analogies in fluid turbulence," Phys. Rev. Lett. 60, 1947 (1988).

${ }^{55}$ A. J. Chorin, "Constrained random walks and vortex filaments in turbulence theory," Commun. Math. Phys. 132, 519 (1990).

${ }^{56}$ L. Mahrt, "Intermittency of atmospheric turbulence," J. Atmos. Sci. 46, 79 (1989).

${ }^{57}$ A. J. Chambers and R. A. Antonia, "Atmospheric estimates of power-law exponents $\mu$ and $\mu_{\theta}$," Boundary Layer Meteorol. 28, 343 (1984).

${ }^{58} \mathrm{R}$. A. Antonia and A. J. Chambers, "On the correlation between turbulent velocity and temperature derivatives in the atmospheric surface layer," Boundary Layer Meteorol. 18, 399 (1980). 